\title{
Real-world use of talimogene laherparepvec in Germany: a retrospective observational study using a prescription database
}

\author{
Karly S Louie*,1, Victoria Banks ${ }^{2}$ (D) Florian Scholz ${ }^{3}$, Hartmut Richter ${ }^{3}$, Katarina Öhrling ${ }^{4}$, \\ Peter Mohr ${ }^{\ddagger}, 5$ \& Sebastian Haferkamp ${ }^{\ddagger}, 6$ \\ ${ }^{1}$ Amgen Ltd, 1 Uxbridge Business Park, Uxbridge, UB8 1DH, UK \\ ${ }^{2}$ VLB Contractors Ltd, 17 Marling Way, Gravesend, DA12 4DW, UK \\ ${ }^{3}$ IQVIA, Real World Insights, Unterschweinstiege 2-14, 60549, Frankfurt, Germany \\ ${ }^{4}$ Amgen Europe, Suurstoffi 22, 6343, Rotkreuz, Switzerland \\ ${ }^{5}$ Elbe-Klinikum, Am Krankenhaus 1, 21614, Buxtehude, Germany \\ ${ }^{6}$ University Hospital Regensburg, Franz-Josef-Strauss-Allee 11, 93053, Regensburg, Germany \\ *Author for correspondence: Tel.: +44 (0)1895 525138; klouie@amgen.com \\ $\ddagger$ Authors are joint senior authors
}

\begin{abstract}
Aim: There is a growing body of data on real-world use of talimogene laherparepvec (T-VEC). We aimed to characterize real-world T-VEC use using a nationally representative German prescription database covering $60 \%$ of prescriptions reimbursed. Patients \& methods: A retrospective analysis was conducted using the German IMS ${ }^{\circledR}$ LRx prescription database, analyzing patients aged $\geq 18$ years with an initial T-VEC prescription at $10^{6}$ plaque-forming units (PFU) $/ \mathrm{ml}$ and $\geq 1$ subsequent prescription at $10^{8} \mathrm{PFU} / \mathrm{ml}$. Median time on T-VEC treatment, patient characteristics and patterns of T-VEC use were described. Results: Of 127 patients prescribed T-VEC, 72 patients $(57 \%)$ met study criteria. About two-thirds of these patients initiated T-VEC in 2017. Median age at T-VEC initiation was 74 years (range: 44 to 91). Most prescriptions (88\%) were dispensed from hospitals. At study end, 26 (36\%) patients remained on T-VEC; 46 (64\%) had ended treatment. Median duration of T-VEC treatment for all patients was 18.7 weeks (95\% Cl: 15.3-26.9) and was longer among those who initiated treatment in 2017 versus 2016 (26.7 vs 15.6 weeks, respectively). Median volume administered for the first $10^{6} \mathrm{PFU} / \mathrm{ml}$ and second $10^{8} \mathrm{PFU} / \mathrm{ml}$ was $4 \mathrm{ml}$; the volume decreased for subsequent administrations $(2 \mathrm{ml}$ by the eighth administration and $1 \mathrm{ml}$ by the 16th administration). Conclusion: This real-world prescription database study showed that patients who initiated treatment in 2017 had a treatment duration in clinical practice that corresponded with the European Summary of Product Characteristics guideline of continuing T-VEC for $\geq 6$ months. Additional long-term data linking drug use with clinical outcomes are needed.
\end{abstract}

First draft submitted: 18 December 2019; Accepted for publication: 24 January 2020; Published online: 13 February 2020

Keywords: $\bullet$ Germany $\bullet$ immunotherapy $\bullet$ melanoma $\bullet$ prescription $\bullet$ real-world $\bullet$ retrospective $\bullet$ talimogene laherparepvec $\bullet T-V E C$

In 2018, there were over 31,000 new cases of melanoma of the skin in Germany and over 3600 deaths, and the age-standardized incidence and mortality rates per 100,000 population were 21.6 and 1.6 , respectively [1]. The survival of patients with unresectable and metastatic melanoma has substantially improved over the last decade with the development of novel systemic immunotherapies, such as anti-CTLA- 4 and anti-PD-1/anti-PD-L1 agents and targeted therapies [2]. Beyond systemic treatment, intratumoral therapies may benefit specific patient groups, for example, those with extensive and recurrent locoregional disease and high tumor burden [3]. Due to a relatively lower risk for toxicity, intratumoral therapies might be a valuable option in elderly patients with co-morbidities, where systemic treatment options are contraindicated [4].

Talimogene laherparepvec (T-VEC) is a first-in-class intratumoral oncolytic immunotherapy derived from herpes simplex virus-1 and genetically engineered to selectively replicate in tumor cells and produce granulocyte-

Future Medicine 
macrophage colony-stimulating factor (GM-CSF) [5,6]. Administration of T-VEC can lead to apoptosis of tumor cells, increased antigen presentation and enhanced antitumor immunity [5,6]. In the Phase III OncoVEX (GMCSF) Pivotal Trial in Melanoma (OPTiM) study, T-VEC demonstrated significantly improved overall response rate, compared with subcutaneous GM-CSF (31.5 vs 5.7\%, respectively), and was well tolerated [7,8]. The effcacy of T-VEC versus GM-CSF was more pronounced in patients with stage IIIB-IVM1a disease than in those with stage IIIB-IVM1c disease. The difference in median overall survival between patients treated with T-VEC versus GM-CSF was 19.6 months longer in those with stage IIIB-IVM1a (41.1 vs 21.5 months) compared with 4.4 months longer in those with stage IIIB-IVM1c (23.3 vs 18.9 months) $[7,9]$. Consistent with this, the overall response rate ( $40.5 \%$ with T-VEC vs $2.3 \%$ with GM-CSF) was higher in patients with stage IIIB-IVM1a disease than in the overall population [7,9]. Consequently, T-VEC was approved in Europe in 2015 for the treatment of adults with unresectable melanoma that is regionally or distantly metastatic (stage IIIB-IVM1a) with no bone, brain, lung or other visceral disease [10].

Evidence from real-world studies extends and complements data obtained from randomized controlled trials, thereby helping to provide a more comprehensive overview of a medication's potential role in everyday clinical practice [11]. Several studies have assessed the effectiveness and safety of T-VEC in real-world settings [12-18]. Across five studies of T-VEC use in routine practice in the USA, the percentage of patients achieving a complete response ranged from 20 to $44 \%$. Four of these studies also reported partial response rates, which ranged from 4 to $18 \%$ [1216]. In addition, one small prospective study of 26 patients in The Netherlands reported a complete response rate of $62 \%$ and a partial response rate of $27 \%$ [17]. T-VEC was generally well tolerated across these studies, with most patients who reported adverse events experiencing mild influenza-like symptoms [12-18].

T-VEC requires repeat administration 3 weeks after the initial dose and every 2 weeks thereafter [10]. T-VEC is available in two doses: $10^{6}$ plaque-forming units $(\mathrm{PFU}) / \mathrm{ml}$ and $10^{8} \mathrm{PFU} / \mathrm{ml}$, both supplied in 1 - $\mathrm{ml}$ single-use vials [10]. The $10^{6} \mathrm{PFU} / \mathrm{ml}$ dose should be used for the first treatment and the $10^{8} \mathrm{PFU} / \mathrm{ml}$ dose for subsequent treatments. The volume to be injected into each lesion is dependent on the size of the lesion, with a maximum injection volume per treatment visit of $4 \mathrm{ml}$ [10]. While real-world data on the effectiveness and safety of T-VEC are gradually accumulating, data on treatment persistence and patterns in clinical practice are still scarce.

The aim of the current study was to provide real-world data on T-VEC use in Germany. Specifically, the objectives were to describe treatment persistence (i.e., estimates of median time on treatment), characteristics of patients receiving T-VEC and patterns relating to T-VEC use (dose and volume).

\section{Patients \& methods}

Study design

This was a retrospective observational cohort study of adult patients prescribed T-VEC in the German IMS ${ }^{\circledR}$ LRx longitudinal prescription database (IQVIA, Frankfurt am Main, Germany). The IMS LRx database collects information from centers nationwide that process prescription data related to patients with statutory health insurance, and contains information on approximately $60 \%$ of all prescriptions reimbursed in Germany [19]. In addition to full prescription information (including date, volume and dose concentration), data on demographics (age, sex and statutory health insurance status) are also collected. The database does not include information on patient diagnoses, laboratory tests or treatment outcomes. The database is fully anonymized and complies with relevant regulations for protecting patient privacy.

The baseline period spanned from 11 July 2011 (the date that the first systemic therapies for melanoma were approved in Germany) to the date of the first $10^{6} \mathrm{PFU} / \mathrm{ml} \mathrm{T-VEC} \mathrm{prescription} \mathrm{(index} \mathrm{date)} \mathrm{for} \mathrm{each} \mathrm{patient}$ (T-VEC was reimbursed in Germany from 15 June 2016). The follow-up period spanned from the index date to the date of discontinuation of T-VEC, transfer out of the IMS LRx database, or the end of the study (31 December 2017), whichever came first (Figure 1).

\section{Patient population}

Patients aged $\geq 18$ years with a T-VEC prescription with a dose concentration of $10^{6} \mathrm{PFU} / \mathrm{ml}$ and at least one subsequent prescription with a dose concentration of $10^{8} \mathrm{PFU} / \mathrm{ml}$ recorded during the study period were included. Patients with only $10^{6} \mathrm{PFU} / \mathrm{ml}$ doses or $10^{8} \mathrm{PFU} / \mathrm{ml}$ doses during the study period, and patients whose first prescription recorded in the IMS LRx database was the $10^{8} \mathrm{PFU} / \mathrm{ml}$ dose were excluded. 


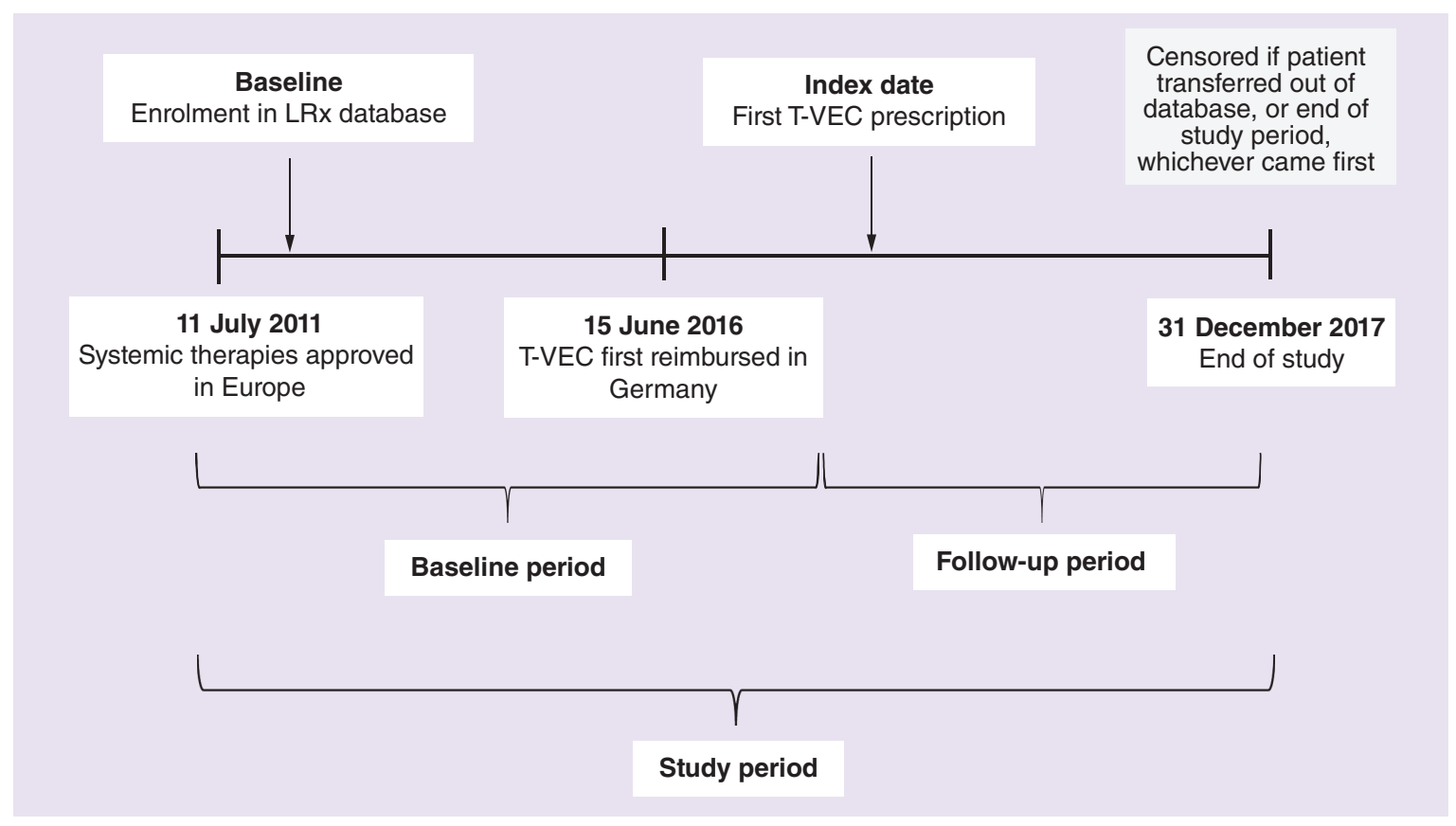

Figure 1. Study design. Deidentified patient data used from the German IMS ${ }^{\circledR}$ LRx database.

LRx: Longitudinal prescription; T-VEC: Talimogene laherparepvec.

\section{Outcome measures}

The primary outcome was T-VEC treatment persistence in the overall population. A persistence analysis stratified by year of T-VEC treatment initiation (2016 or 2017) was also performed. Secondary outcomes were the demographics of people receiving T-VEC, dose concentration and volume of T-VEC used, treatment patterns (discontinuation, interruption and reinitiation of treatment) and other melanoma therapies prior to and after T-VEC treatment.

For the purposes of treatment persistence analyses, patients were defined as having ongoing treatment if their last prescription extended beyond the end of the study period, or did not extend beyond the end of the study period but the gap between the last prescription and the end of the study period was $\leq 42$ days. The estimation of treatment persistence allowed for 42 days between prescriptions based on a recommended 14-day interval between $10^{8} \mathrm{PFU} / \mathrm{ml}$ doses plus a 28-day permissible gap after the last dose was administered in which a patient could go without a dose, in accordance with the product label and established methodology for persistence analyses [10,20]. Patients whose last prescription was more than 42 days prior to end of the study period were defined as having ended treatment. For patients who ended treatment, 14 days were added to the treatment persistence calculation to allow for the recommended gap between $10^{8} \mathrm{PFU} / \mathrm{ml}$ doses [10]. Treatment duration in weeks was calculated as follows:

- Patients with ongoing treatment: ([Study end date - date of first prescription] +1$) / 7$

- Patients who ended treatment: ([Date of last prescription - date of first prescription +14$]+1) / 7$

For treatment pattern analyses, a treatment interruption was defined as a period of more than 28 days between the first and second prescription (relating to the 3-week dosing interval between the priming dose and second dose and a permissible gap of 7 days [10]), and a period of more than 21 days between subsequent prescriptions (relating to the 2 -week interval between doses after the priming dose and a permissible gap of 7 days [10]). Reasons for ending treatment are not recorded in this prescription database.

For the description of treatment before and after T-VEC treatment, patients had to have at least 1 year of follow-up time in the IMS LRx prescription database. 
Patients with at least one

T-VE prescription from

15 June 2016 to 31 December 2017

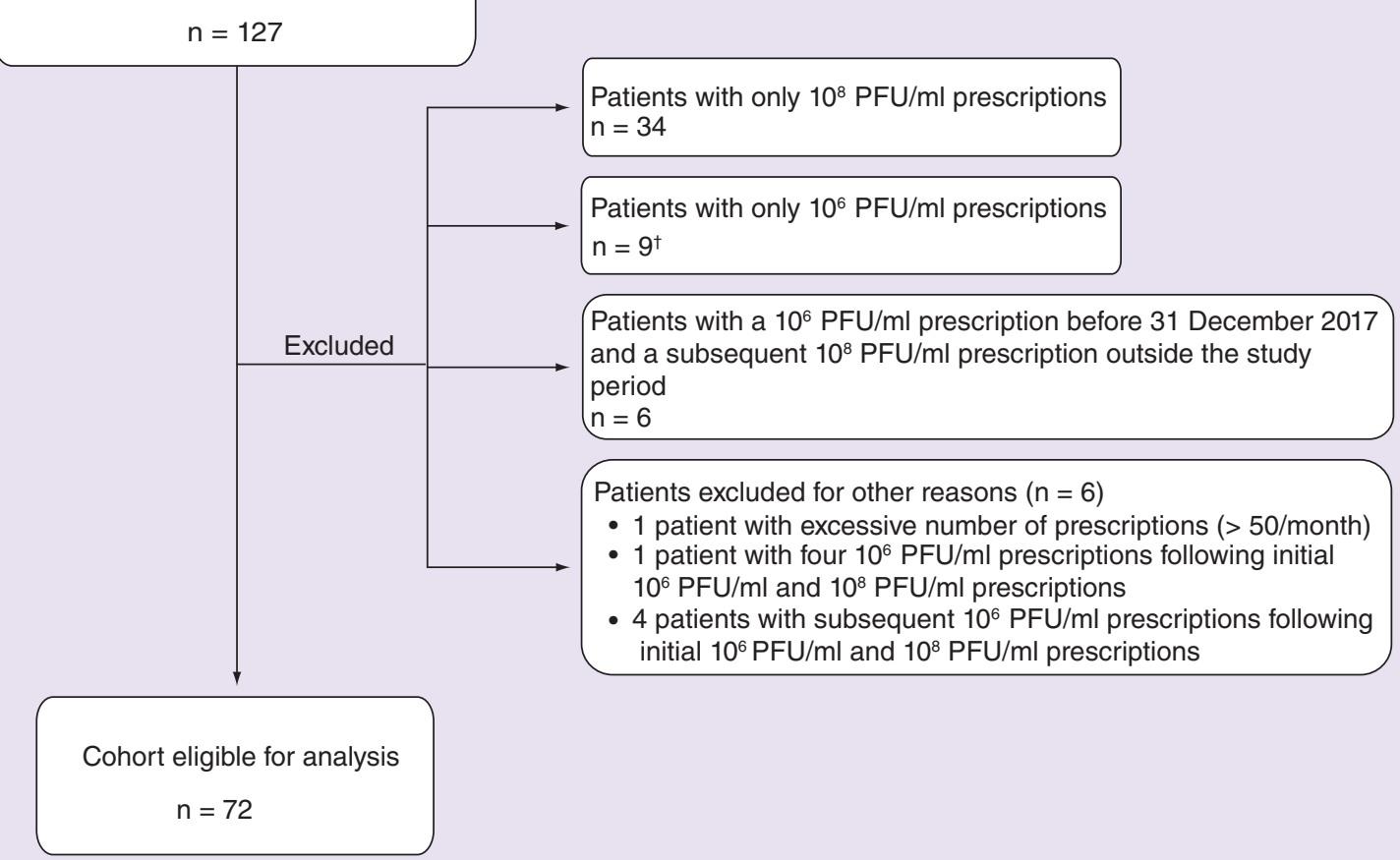

Figure 2. Patient flow.

tOf the nine patients excluded, eight had only a single $10^{6} \mathrm{PFU} / \mathrm{ml}$ prescription and one had multiple $10^{6} \mathrm{PFU} / \mathrm{ml}$ prescriptions.

PFU: Plaque-forming unit; T-VEC: Talimogene laherparepvec.

\section{Statistical analyses}

Treatment persistence was assessed using Kaplan-Meier survival analyses and descriptive statistics. For KaplanMeier analyses, patients were censored if they transferred out of the database, or if the end of the study period was reached, whichever came first.

Summary statistics (mean, standard deviation [SD], median, quartiles and range for continuous variables, and numbers and percentages for categorical variables) are provided for the secondary outcomes.

\section{Results}

\section{Study population}

In total, 127 patients from the IMS LRx database were prescribed T-VEC during the study period. Of these, 72 (57\%) met study criteria (Figure 2). The median age was 74 years (Table 1). Of the 32 patients who had their sex recorded in the database, slightly more than half $(56 \%)$ were male. Patients with T-VEC prescriptions recorded in the IMS LRx database were primarily from the South $(35 / 72,49 \%)$ and West $(21 / 72,29 \%)$ regions of Germany. The majority of patients had their T-VEC dispensed from hospital pharmacies. Two-thirds of patients (44/72, 61\%) initiated T-VEC therapy in 2017.

\section{Treatment patterns}

At the end of the study, 46 patients (64\%) had ended T-VEC treatment and 26 patients (36\%) had ongoing T-VEC therapy (Table 1). Figure 3 shows the individual patient treatment patterns from T-VEC initiation to end of study. Twelve patients had treatment interruptions ( $>28$ days between prescriptions). Among these 12 patients with a treatment interruption, nearly all had one treatment interruption (with the exception of one patient who 
Table 1. Study population characteristics.

\begin{tabular}{|ll|}
\hline Characteristic & Overall population $(\mathbf{n = 7 2})$ \\
\hline Sex: & $74(66-81)[44-91]$ \\
- Male & $18(25)$ \\
- Female & $14(19)$ \\
- Unknown & $40(56)$ \\
\hline Area of Germany: & \\
- North & $11(15)$ \\
- West & $21(29)$ \\
- East & $5(7)$ \\
- South & $35(49)$ \\
\hline Dispensing pharmacy: & $63(88)$ \\
- Hospital & $9(12)$ \\
- Retail & \\
\hline Year of T-VEC initiation: & $28(39)$ \\
- 2016 & $44(61)$ \\
- 2017 & \\
\hline Status at the end of study: & $46(64)$ \\
- Ended T-VEC treatment & $26(36)$ \\
\hline
\end{tabular}

Data represented as $n$ (\%) unless otherwise stated.

IQR: Interquartile range; T-VEC: Talimogene laherparepvec

had two treatment interruptions). The median time to first T-VEC dose interruption was 12.4 weeks (range: 4.9 to 28.0 weeks). Treatment interruption lasted between 4.1 and 8.0 weeks for the first interruption before T-VEC treatment was reinitiated. For the patient who had two treatment interruptions, the first and second interruption lasted 6 and 10 weeks, respectively. Of the 13 interruptions that occurred, $77 \%$ had a treatment interruption of $\leq 6$ weeks.

\section{Treatment persistence}

The median duration of T-VEC treatment by Kaplan-Meier estimation for all patients was 18.7 weeks (95\% CI: 15.3-26.9), with a longer duration among those who initiated treatment in 2017 compared with those who initiated treatment in 2016 (median: 26.7 vs 15.6 weeks; Figure 4).

Among those who ended T-VEC treatment during the study period, the mean and median duration of treatment were 19.0 (SD: 12.3 ) and 15.1 weeks (interquartile range: 9.9 to 23.7 , range: 5.3 to 60.9 ), respectively.

\section{Dose \& volume}

The mean and median cumulative number of vials were 19.63 (SD: 14.71) and 15 (range: 3 to 64), respectively. Among patients who discontinued treatment, the mean and median cumulative number of vials were 20.61 (SD: 15.61) and 16 (range: 3 to 64); and for patients with treatment ongoing, the mean and median were 25.5 (SD: 14.36) and 25 (range: 6 to 52), respectively. The median volume administered for the first $10^{6} \mathrm{PFU} / \mathrm{ml}$ and second $10^{8} \mathrm{PFU} / \mathrm{ml} \mathrm{T-VEC} \mathrm{dose} \mathrm{administrations} \mathrm{was} 4 \mathrm{ml}$. Generally, the volume prescribed decreased from the initiation administration to subsequent administrations (Figure 5). Five patients had a record of one or more prescriptions for a volume of more than $4 \mathrm{ml}$.

The median volume administered for all $10^{6} \mathrm{PFU} / \mathrm{ml}$ prescriptions was $4 \mathrm{ml}$, and median for all $10^{8} \mathrm{PFU} / \mathrm{ml}$ prescriptions was $2 \mathrm{ml}$. In total, the median cumulative number of vials per patient was 15 (interquartile range: 10 to 27 , range: 3 to 64 ).

\section{Other melanoma treatments}

18 patients (25\%) had at least 1 year of follow-up in the IMS LRx database to characterize treatment received before, during and after T-VEC (Table 2). Table 3 shows the treatment sequencing for each individual patient. A third of patients $(6 / 18,33 \%)$ received T-VEC as first-line therapy, with 12 patients $(67 \%)$ having received prior therapy before T-VEC. More than half of patients (10/18) received T-VEC as second-line treatment. The most common therapy received prior to T-VEC was pembrolizumab followed by ipilimumab, nivolumab, imiquimod and IFN- $\alpha-2$ B. Four patients (22\%) received combination treatment with T-VEC: two patients received T-VEC plus pembrolizumab and one patient each received T-VEC plus ipilimumab and T-VEC plus imiquimod. Of 
Table 2. Systemic treatment before, during and after talimogene laherparepvec treatment in 18 patients with adequate follow-up of $\geq 1$ year in the German IMS ${ }^{\circledR}$ LRx database.

\begin{tabular}{|c|c|}
\hline Treatmeny before T-VEC & $\mathrm{n}=18$ \\
\hline Patients who received treatment before T-VEC treatment ${ }^{\dagger}$ : & $12(67)$ \\
\hline - Pembrolizumab & $6(50)$ \\
\hline - Nivolumab & $2(17)$ \\
\hline - Imiquimod & $2(17)$ \\
\hline \multicolumn{2}{|l|}{ Treatment during T-VEC } \\
\hline Patients treated in combination with T-VEC: & $4(22)$ \\
\hline - Pembrolizumab & $2(11)$ \\
\hline - Ipilimumab & $1(6)$ \\
\hline - Pembrolizumab & $2(14)$ \\
\hline - Ipilimumab§ & $1(7)$ \\
\hline - Ipilimumab + nivolumab $\mathbb{I}$ & $1(7)$ \\
\hline - Imiquimod\# & $1(7)$ \\
\hline \multicolumn{2}{|c|}{$\begin{array}{l}\text { Data represented as } n(\%) \text { unless otherwise stated. } \\
\dagger \text { Patients may have received more than one line of prior therapy. } \\
\text { ¥Pembrolizumab treatment was prescribed } 15 \text { days after the last T-VEC prescription for both patients. } \\
\text { §Patient was initiated on ipilimumab on the same day as the last day of T-VEC treatment. } \\
\mathbb{I} \text { Ipilimumab plus nivolumab treatment was prescribed } 55 \text { weeks after the last T-VEC prescription. } \\
\text { \#Patient was prescribed imiquimod on the same day as the last T-VEC prescription. } \\
\text { LRx: Longitudinal prescription; T-VEC: Talimogene laherparepvec. }\end{array}$} \\
\hline
\end{tabular}

\begin{tabular}{|c|c|c|c|c|c|}
\hline Patient number & First-line & Second-line & Third-line & Fourth-line & Fifth-line \\
\hline 1 & T-VEC & & & & \\
\hline 2 & T-VEC & & & & \\
\hline 3 & $\mathrm{~T}^{-V E C^{\dagger}}$ & & & & \\
\hline 4 & $\mathrm{~T}^{-\mathrm{VEC}}{ }^{\dagger}$ & & & & \\
\hline 5 & $\mathrm{~T}^{-\mathrm{VEC}}{ }^{\dagger}$ & & & & \\
\hline 6 & $\mathrm{~T}^{-\mathrm{VEC}^{\dagger}}$ & & & & \\
\hline 7 & IFN- $\alpha-2 B$ & T-VEC & & & \\
\hline 8 & Imiquimod & T-VEC + imiquimod & & & \\
\hline 9 & Imiquimod $^{\dagger}$ & T-VEC $\dagger$ & Imiquimod $^{\dagger}$ & & \\
\hline 10 & Pembrolizumab $^{\dagger}$ & $\mathrm{T}^{\mathrm{V} V \mathrm{C}}+$ pembrolizumab $^{\dagger}$ & & & \\
\hline 11 & Pembrolizumab $^{\dagger}$ & T-VEC $\dagger$ & & & \\
\hline 12 & Pembrolizumab $^{\dagger}$ & T-VEC + pembrolizumab ${ }^{\dagger}$ & Pembrolizumab $^{\dagger}$ & & \\
\hline 13 & Pembrolizumab $^{\dagger}$ & $\mathrm{T}^{-V E C^{\dagger}}$ & & & \\
\hline 14 & Ipilimumab ${ }^{\dagger}$ & $\mathrm{T}^{-V E C^{\dagger}}$ & Pembrolizumab $^{\dagger}$ & & \\
\hline 15 & Nivolumab $^{\dagger}$ & T-VEC ${ }^{\dagger}$ & & & \\
\hline 16 & Nivolumab $^{\dagger}$ & $\mathrm{T}^{-V E C^{\dagger}}$ & & & \\
\hline 17 & Ipilimumab $^{\dagger}$ & Pembrolizumab $^{\dagger}$ & $\mathrm{T}^{-V E C^{\dagger}}$ & Ipilimumab + nivolumab ${ }^{\dagger}$ & \\
\hline 18 & Ipilimumab $^{\dagger}$ & Pembrolizumab $^{\dagger}$ & Ipilimumab $^{\dagger}$ & 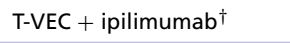 & Ipilimumab ${ }^{\dagger}$ \\
\hline
\end{tabular}




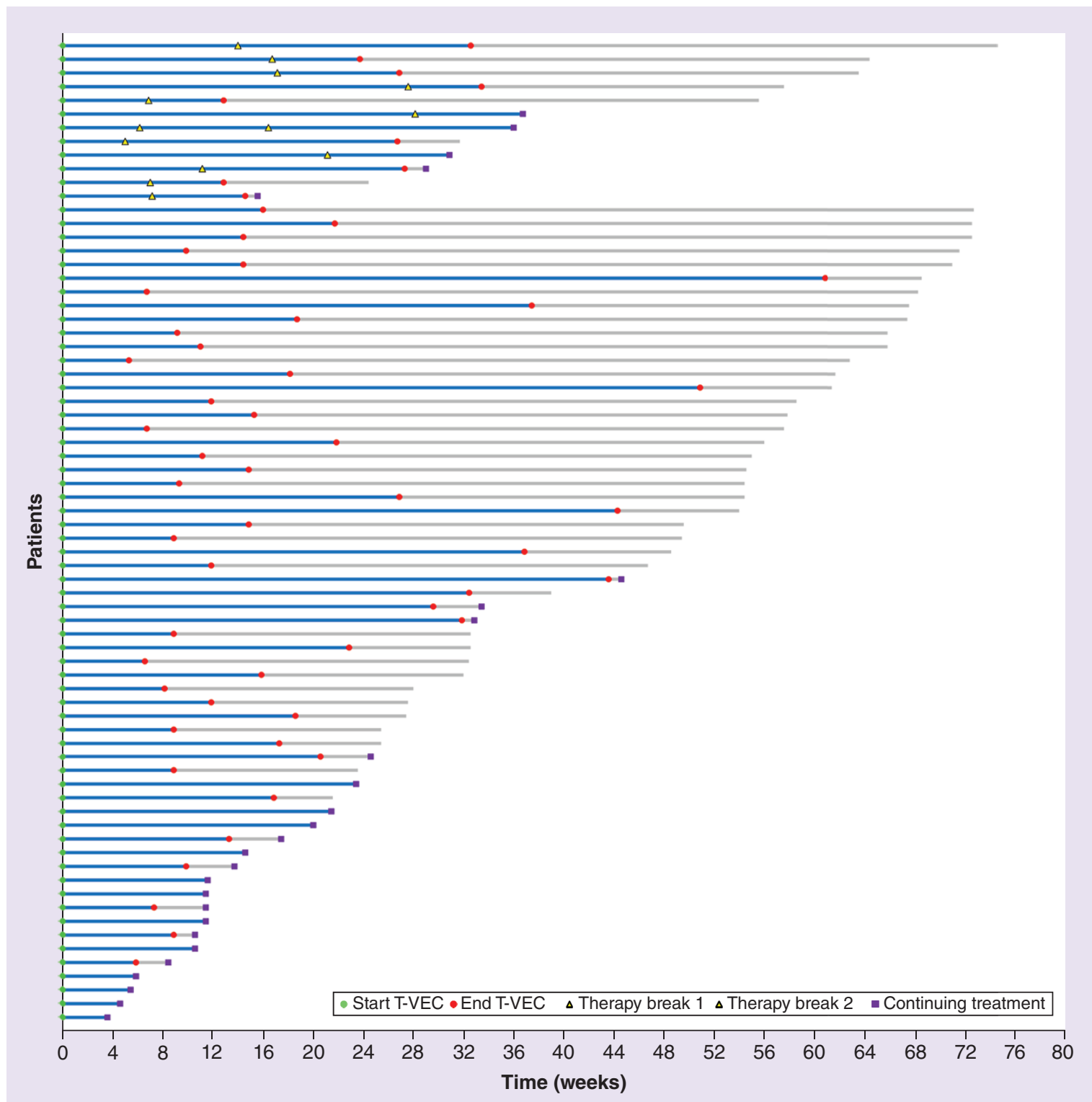

Figure 3. Swimmer plot showing individual patient treatment history.

T-VEC: Talimogene laherparepvec.

the 14 patients who discontinued T-VEC treatment, five patients (36\%) received further treatment after T-VEC: two patients received pembrolizumab, one patient each received ipilimumab, ipilimumab plus nivolumab, and imiquimod. For those two patients who received pembrolizumab, both were prescribed pembrolizumab 15 days after the last T-VEC prescription. Patients who received ipilimumab and imiquimod after T-VEC were prescribed these on the same day as the last T-VEC prescription. The patient prescribed with ipilimumab plus nivolumab received this combination 55 weeks after the last T-VEC prescription. Among the six patients who received TVEC as first-line treatment, four patients had discontinued T-VEC treatment and did not receive any subsequent second-line therapy.

\section{Discussion}

In this retrospective observational cohort study of 72 adult patients included in the German IMS LRx database who were prescribed T-VEC from 15 June 2016 (the date of the drug's launch in Germany) to 31 December 2017, approximately a third of patients (36\%) remained on T-VEC at the end of the study, and two-thirds (64\%) had 


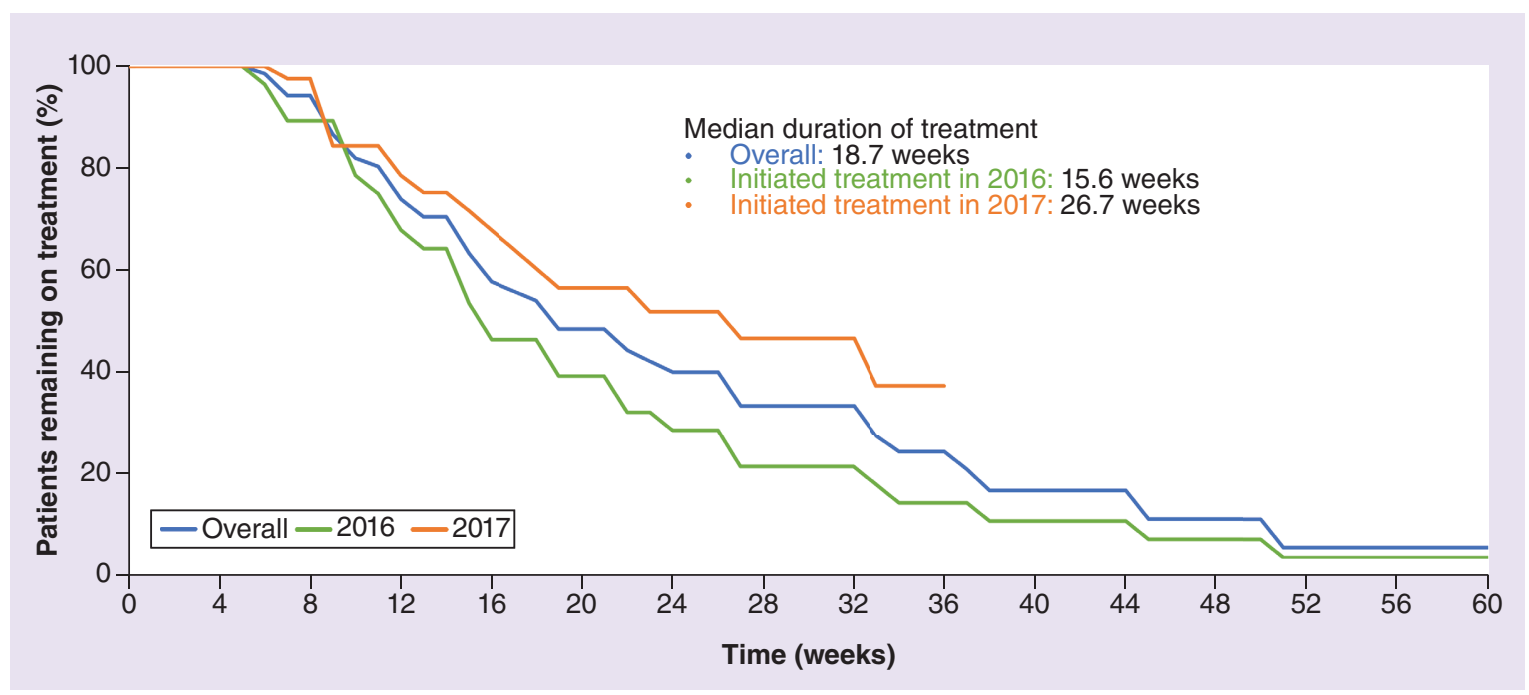

Figure 4. Talimogene laherparepvec treatment persistence, overall and by year of treatment initiation (Kaplan-Meier estimation).

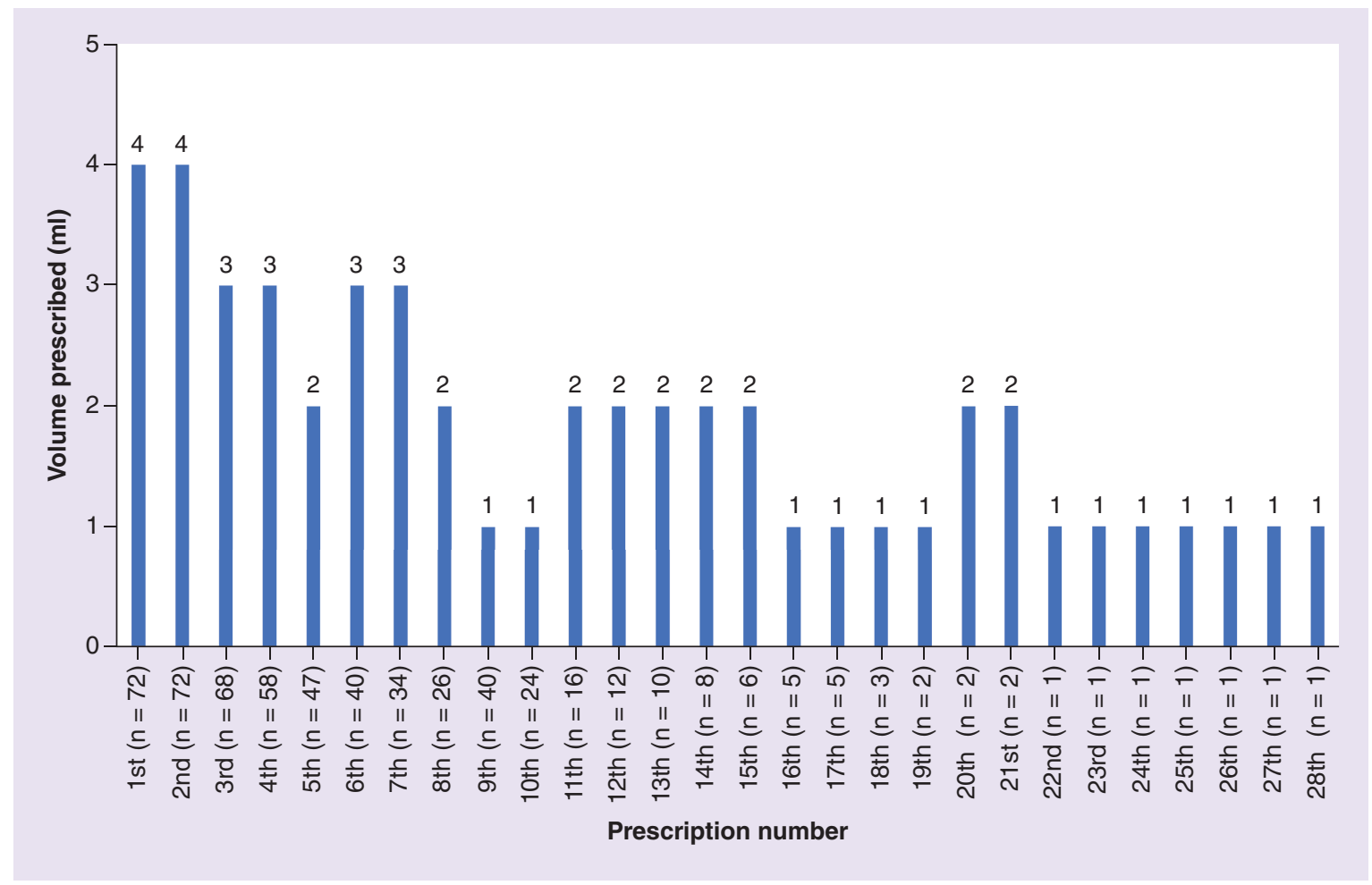

Figure 5. Median talimogene laherparepvec volume prescribed for each prescription. The first prescription was for $10^{6} \mathrm{PFU} / \mathrm{ml}$ and subsequent prescriptions were for $10^{8} \mathrm{PFU} / \mathrm{ml}$.

stopped treatment. The median duration of T-VEC treatment was 18.7 weeks; when stratified by year of treatment initiation, median treatment duration was longer among those who initiated treatment in 2017 (26.7 weeks) versus 2016 (15.6 weeks). The increase in treatment duration from 2016 to 2017 may reflect differences in patient selection following drug launch and early experience with T-VEC use. These treatment patterns are likely to change over time as physicians gain experience with the treatment. It is notable that, for patients who initiated treatment in 2017, the duration of treatment was broadly consistent with the median duration of T-VEC treatment among 
patients with stage IIIB-IVM1a disease included in the pivotal Phase III OPTiM study (25.7 weeks) [9]. The results were also consistent with the European Summary of Product Characteristics recommendation to continue T-VEC for at least 6 months unless other treatment is required or until there are no injectable lesions to treat [10]. Only 12 patients $(17 \%)$ had T-VEC dose interruptions and most patients had an interruption $\leq 6$ weeks.

Real-world use of T-VEC in German patients with stage IIIB-IVM1a melanoma has previously been assessed in a retrospective chart review of 27 patients who started T-VEC treatment between June 2016 and July 2017 [21]. In that study, the median duration of T-VEC treatment was 22.1 weeks, although longer in patients with stage IIIB/C disease than in those with IVM1a disease (27.9 vs 22.0 weeks) [21]. Treatment duration was also longer in patients who received T-VEC as first-line therapy (23.1 weeks) and second-line therapy (22.1 weeks) than in those who received it as third-line or later therapy (15.9 weeks) [21]. A chart review of 62 patients in Austria and Switzerland reported that patients with stage IIIB/C disease received a median of 11 doses of T-VEC [22], which, taking into account recommended T-VEC dosing schedules, is in line with our results. Two other studies in clinical practice settings in the USA have reported shorter treatment durations with T-VEC than we observed in our current study and may be explained by the lower tumor load burden in these patients $[15,18]$. However, these US studies also included more advanced patients with stage IV disease. This reflects the differences in approved indication between the US (unresectable cutaneous, subcutaneous and nodal lesions in patients with melanoma recurrent after initial surgery [stage IIIB-IV] [23]) and Europe (unresectable melanoma that is regionally or distantly metastatic with no bone, brain, lung or other visceral disease [stage IIIB, IIIC and IVM1a] [10]).

18 patients had long-term follow-up data available. In this small subset, T-VEC was most commonly prescribed in the second-line (with pembrolizumab being the most common first-line treatment choice). Seven of the 18 patients received pembrolizumab at some point in their treatment sequence (either before, during or after T-VEC treatment). Only a third received T-VEC as first-line therapy, which is in contrast to the previously mentioned German chart review, in which around two-thirds of patients received first-line T-VEC [21]. For four patients who received T-VEC as first-line therapy and discontinued treatment, there was no record of subsequent therapy, potentially suggesting the effectiveness of T-VEC when used earlier to treat patients.

The recommended dosing volume for T-VEC is up to $4 \mathrm{ml}$ maximum per administration visit and is dependent on lesion size [10]. In the current study, the median volume of T-VEC administered was $4 \mathrm{ml}$ for the first $10^{6}$ $\mathrm{PFU} / \mathrm{ml}$ dose and second $10^{8} \mathrm{PFU} / \mathrm{ml}$ dose, decreasing to $2 \mathrm{ml}$ by the eighth administration and to $1 \mathrm{ml}$ by the 16th administration (and for most subsequent doses). Lesion sizes were not recorded in the current study. As the initial volume (first injection of $10^{6} \mathrm{PFU} / \mathrm{ml}$ ) was $4 \mathrm{ml}$, this implies that the tumor volume for the study patients was large enough to require the maximum dose; and the decrease in T-VEC volumes prescribed over time may reflect decreasing tumor size or number, and hence a potential treatment effect. Decreases in prescribed T-VEC volumes were consistent with findings in the German retrospective chart review [21] and in other clinical practice studies $[12-15,17]$.

The median age of patients included in the current study (74 years) was substantially higher than that of patients with stage IIIB-IVM1a disease included in the OPTiM trial (63 years) [9]. Similarly, patients included in the German retrospective chart review were older than in the OPTiM trial (median age 68 years) [21]. These differences may reflect the fact that patients treated in routine clinical practice, particularly the elderly, are more likely to have co-morbidities and other clinical characteristics that exclude them from participating in clinical trials where there are strict inclusion and exclusion criteria. These results highlight the importance of real-world studies in complementing evidence from clinical trials [11,24].

The study has several strengths. One strength is the rapid availability of data with large patient pools from the whole German population for analysis, which is time and cost-efficient as compared with conducting a primary data collection study. As the German IMS LRx database covers $60 \%$ of all prescriptions in Germany, these data are broadly generalizable to the T-VEC population prescribed in Germany over the study period. Also, the majority of patients had T-VEC dispensed from hospital pharmacies which accurately reflects the requirement for T-VEC to be administered by a qualified physician that has completed training through the controlled distribution program [9]. As T-VEC requires special handling in terms of potential perceived risks as an advanced therapy medicinal product and due to cold-chain requirements, T-VEC is mainly dispensed via hospital pharmacies. For a minority of patients, T-VEC may be dispensed from retail pharmacy (e.g., community pharmacy) but would be located in close proximity to the prescriber's office-based practices/clinic. However, the study has acknowledged limitations. First, the database used is a prescription database, and does not contain information on diagnosis (e.g., to confirm stage of disease), therapeutic outcomes, co-morbidities, safety (e.g., adverse drug reactions), laboratory parameters 
(e.g., to confirm $B R A F$ status) or reasons for stopping treatment. Consequently, it was not possible to control for potential confounding factors that might have had an impact on treatment persistence, for example, severity of disease. Second, treatment persistence was calculated based on prescription data only, which may not reflect what was actually administered in practice.

Despite these limitations, this study provides valuable information regarding the real-world use of T-VEC in Germany. Patients were older in the real-world analysis compared with those who participated in clinical trials, and over $80 \%$ of patients were treated with T-VEC without dose interruptions. The median duration of treatment in patients prescribed T-VEC during the latter part of the study (2017) was consistent with licensed recommendations, as were the median dose volumes administered throughout the study. Among patients with adequate follow-up time to describe treatment patterns, a third of patients received T-VEC as first-line therapy. This study characterizes the first patients treated with T-VEC in Germany. Additional long-term studies in which drug utilization data are linked with patient clinical data are needed to assess the effectiveness of T-VEC over time in the real-world setting.

\section{Conclusion}

This real-world prescription database study highlights that German patients who initiated treatment in 2017 had a median duration of treatment in routine clinical practice that corresponded with the European Summary of Product Characteristics guideline of continuing T-VEC for at least 6 months.

\section{Summary points}

- The objective of this study was to characterize real-world talimogene laherparepvec (T-VEC) use.

- This was a retrospective cohort study, which used the German IMS ${ }^{\circledR}$ LRx prescription database, which covers $60 \%$ of all prescriptions reimbursed in Germany.

- Patients aged $\geq 18$ years with an initial T-VEC prescription at $10^{6}$ plaque-forming units (PFU)/ml and $\geq 1$ subsequent prescription at $10^{8} \mathrm{PFU} / \mathrm{ml}$ during the study period were included.

- A total of 72 patients met study criteria; about two-thirds of these initiated T-VEC in 2017.

- Median age at T-VEC initiation was 74 years.

- The majority of prescriptions $(88 \%)$ were dispensed from hospitals rather than retail pharmacies.

- The median duration of T-VEC treatment for all patients was 18.7 weeks (95\% Cl: 15.3-26.9).

- T-VEC treatment duration was longer among those who initiated treatment in 2017 versus 2016 (26.7 vs 15.6 weeks, respectively).

- The median volume administered for the first $10^{6} \mathrm{PFU} / \mathrm{ml}$ and second $10^{8} \mathrm{PFU} / \mathrm{ml}$ was $4 \mathrm{ml}$; the volume decreased for subsequent administrations, reducing to $2 \mathrm{ml}$ by the eighth administration and $1 \mathrm{ml}$ by the 16th administration.

- This real-world prescription database study showed that patients who initiated treatment in 2017 had a treatment duration in clinical practice that corresponded with the European Summary of Product Characteristics guideline of continuing T-VEC for $\geq 6$ months.

\section{Author contributions}

KS Louie was responsible for the concept and design of the study. V Banks, F Scholz and H Richter were responsible for acquisition of the data. KS Louie, V Banks, F Scholz, H Richter, and K Öhrling were responsible for the analysis of the data. All the authors were responsible for interpretation of the data, and drafting or revising the manuscript. All the authors gave their final approval of the version to be published and agree to be accountable for all aspects of the work in ensuring that questions related to the accuracy or integrity of any part of the work are appropriately investigated and resolved.

\section{Financial \& competing interest disclosure}

This study was funded by Amgen Inc. (Thousand Oaks, CA, USA). KS Louie and K Öhrling are stockholders and employees of Amgen Inc. $\vee$ Banks is an independent contractor providing epidemiological services to Amgen Inc. F Scholz and $\mathrm{H}$ Richter are employed by IQVIA, Real World Insights. P Mohr has received research grants from Bristol-Myers Squibb (BMS) and Merck Sharp and Dohme (MSD), and consulting fees from Amgen, BMS, GlaxoSmithKline, Merck Serono, MSD, Novartis, Pierre Fabre and Roche. S Haferkamp has received research grants from BMS and Novartis, and consulting fees from Amgen, BMS, Novartis and Roche. The authors have no other relevant affiliations or financial involvement with any organization or entity with a financial interest in or financial conflict with the subject matter or materials discussed in the manuscript apart from those disclosed. 
Medical writing support (including development of a first draft and subsequent drafts in consultation with the authors, assembling tables and figures, collating author comments, copyediting, fact checking and referencing) was provided by Emma McConnell at Aspire Scientific Ltd (Bollington, UK), and funded by Amgen Inc. (CA, USA).

Ethical conduct of research

The German IMS ${ }^{\circledR}$ LRx longitudinal prescription database (IQVIA, Frankfurt am Main, Germany) used in this study is fully anonymized and complies with relevant regulations for protecting patient privacy.

\section{Open access}

This work is licensed under the Attribution-NonCommercial-NoDerivatives 4.0 Unported License. To view a copy of this license, visit http://creativecommons.org/licenses/by-nc-nd/4.0/

\section{References}

Papers of special note have been highlighted as: • of interest; $\bullet \bullet$ of considerable interest

1. Globocan. Cancer fact sheet: Germany (2018). https://gco.iarc.fr/today/data/factsheets/populations/276-germany-fact-sheets.pdf

2. Ascierto PA, Flaherty K, Goff S. Emerging strategies in systemic therapy for the treatment of melanoma. Am. Soc. Clin. Oncol. Educ. Book. 38, 751-758 (2018).

- A useful review article discussing new strategies being investigated for the treatment of melanoma.

3. Wang DY, Johnson DB. Advances in the development of intralesional therapies for melanoma. Melanoma Manag. 3(4), 259-266 (2016).

4. Harrington KJ, Puzanov I, Hecht JR et al. Clinical development of talimogene laherparepvec (T-VEC): a modified herpes simplex virus type-1-derived oncolytic immunotherapy. Expert Rev. Anticancer Ther. 15(12), 1389-1403 (2015).

- This expert review provides a useful overview of intralesional therapies, focusing on talimogene laherparepvec (T-VEC).

5. Liu BL, Robinson M, Han ZQ et al. ICP34.5 deleted herpes simplex virus with enhanced oncolytic, immune stimulating, and anti-tumour properties. Gene Ther. 10(4), 292-303 (2003).

6. Rehman H, Silk AW, Kane MP, Kaufman HL. Into the clinic: talimogene laherparepvec (T-VEC), a first-in-class intratumoral oncolytic viral therapy. J. Immunother. Cancer. 4, 53 (2016).

7. Andtbacka RH, Kaufman HL, Collichio F et al. Talimogene laherparepvec improves durable response rate in patients with advanced melanoma. J. Clin. Oncol. 33(25), 2780-2788 (2015).

•• Reports the primary results of the pivotal OPTiM trial, of T-VEC versus granulocyte-macrophage colony-stimulating factor in patients with unresectable melanoma.

8. Andtbacka RHI, Amatruda T, Nemunaitis J et al. Biodistribution, shedding, and transmissibility of the oncolytic virus talimogene laherparepvec in patients with melanoma. EBioMedicine. 47, 89-97 (2019).

9. Harrington KJ, Andtbacka RH, Collichio F et al. Efficacy and safety of talimogene laherparepvec versus granulocyte-macrophage colony-stimulating factor in patients with stage IIIB/C and IVM1a melanoma: subanalysis of the Phase III OPTiM trial. Onco. Targets Ther. 9, 7081-7093 (2016).

•• Reports a subanalysis of the pivotal OPTiM trial, of T-VEC vs granulocyte-macrophage colony-stimulating factor in patients with stage IIIB-IVM1a unresectable melanoma.

10. European Medicines Agency. Imlygic ${ }^{\circledR}$ (talimogene laherparepvec) summary of product characteristics (2019). www.ema.europa.eu/en/documents/product-information/imlygic-epar-product-information_en.pdf

11. Katkade VB, Sanders KN, Zou KH. Real world data: an opportunity to supplement existing evidence for the use of long-established medicines in health care decision making. J. Multidiscip. Healthc. 11, 295-304 (2018).

- This review paper gives an overview of the importance of real-world evidence to supplement clinical trial data.

12. Louie RJ, Perez MC, Jajja MR et al. Real-world outcomes of talimogene laherparepvec therapy: a multi-institutional experience. J. Am. Coll. Surg. 228(4), 644-649 (2019).

13. Masoud SJ, Hu JB, Beasley GM, Stewart JHT, Mosca PJ. Efficacy of talimogene laherparepvec (T-VEC) therapy in patients with in-transit melanoma metastasis decreases with increasing lesion size. Ann. Surg. Oncol. 26(13), 4633-4641 (2019).

14. Perez MC, Miura JT, Naqvi SMH et al. Talimogene laherparepvec (TVEC) for the treatment of advanced melanoma: a single-institution experience. Ann. Surg. Oncol. 25(13), 3960-3965 (2018).

15. Perez MC, Zager JS, Amatruda T et al. Observational study of talimogene laherparepvec use for melanoma in clinical practice in the United States (COSMUS-1). Melanoma Manag. 6(2), MMT19 (2019).

16. Zhou AY, Wang DY, Mckee S et al. Correlates of response and outcomes with talimogene laherperpvec. J. Surg. Oncol. 120(3), 558-564 (2019).

17. Franke V, Berger DMS, Klop WMC et al. High response rates for T-VEC in early metastatic melanoma (stage IIIB/C-IVM1a). Int. J. Cancer. 145(4), 974-978 (2019). 
18. Chesney J, Awasthi S, Curti B et al. Phase IIIb safety results from an expanded-access protocol of talimogene laherparepvec for patients with unresected, stage IIIB-IVM1c melanoma. Melanoma Res. 28(1), 44-51 (2018).

- Reports the results of a Phase IIIb expanded access protocol of T-VEC for patients with unresected stage IIIB-IVM1c melanoma.

19. Richter H, Dombrowski S, Hamer H, Hadji P, Kostev K. Use of a German longitudinal prescription database (LRx) in pharmacoepidemiology. Ger. Med. Sci. 13, Doc14 (2015).

20. Cramer JA, Roy A, Burrell A et al. Medication compliance and persistence: terminology and definitions. Value Health. 11(1), 44-47 (2008).

21. Mohr P, Haferkamp S, Pinter A et al. Real-world use of talimogene laherparepvec in German patients with stage IIIB to IVM1a melanoma: a retrospective chart review and physician survey. Adv. Ther. 36(1), 101-117 (2019).

22. Hoeller C, Ressler JM, Karasek M et al. Real life use of talimogene laherparepvec in melanoma in centers in Austria and Switzerland. Ann. Oncol. 30(Suppl. 5), 1343P (2019).

23. US FDA. IMLYGIC ${ }^{\circledR}$ (talimogene laherparepvec) package insert (2019). www.fda.gov/media/94129/download

24. Brown ML, Gersh BJ, Holmes DR, Bailey KR, Sundt TM 3rd. From randomized trials to registry studies: translating data into clinical information. Nat. Clin. Pract. Cardiovasc. Med. 5(10), 613-620 (2008). 